\title{
The use of drugs in patients who have undergone bariatric surgery
}

This article was published in the following Dove Press journal:

International Journal of General Medicine

14 May 2014

Number of times this article has been viewed

\section{Mariana de Sousa Prado \\ Geraldo' \\ Fernando Luiz Affonso \\ Fonseca ${ }^{2,3}$ \\ Marisa Regina de Fatima \\ Veiga Gouveia ${ }^{4}$ \\ David Feder ${ }^{4}$}

'Department of Medicine, Faculdade de Medicina do ABC, ${ }^{2}$ Department of Pharmacy and Biochemistry, Universidade Federal de Sao Paulo, ${ }^{3}$ Department of Hematology, Faculdade de Medicina do $A B C$, ${ }^{4}$ Department of Pharmacology, Faculdade de Medicina do ABC, Santo Andre, SP, Brazil
Correspondence: David Feder Principe de Gales Av 821, Santo André, SP 09060-650, Brazil

Tel +55 II 49935000

Email marianaspg@hotmail.com
Abstract: According to the World Health Organization, obesity has become an epidemic in the 21 st century affecting around 300 million people of all ages worldwide. Clinical treatment modalities for this disease are limited and ineffective when it comes to morbidly obese patients (body mass index - the weight in kilograms divided by height in meters squared surpasses $40 \mathrm{~kg} / \mathrm{m}^{2}$ ). Therefore, the alternative, surgical treatment, is the best option for these patients, namely gastric restrictive procedures or an intestinal bypass culminating in a malabsorptive syndrome. In the past 20 years, there has been a $70 \%$ increase in the number of bariatric procedures all over the world. The main pharmacokinetic consequence observed in the postoperative period of these individuals is a higher or lower absorption of orally administered drugs.

Keywords: anatomophysiological alterations, pharmacokinetic, obesity

\section{Introduction}

Researchers all over the world have classified obesity, according to the World Health Organization, as a global epidemic disease of the 21 st century that must be dealt with more effectively in view of the high rates among children and the detrimental consequences in the obese person's health as a whole. ${ }^{1}$ The number of bariatric procedures performed worldwide has increased $70 \%$ in the last 2 decades and the estimated number of annual surgeries is approximately $350,000 .^{2}$

A patient is considered obese if their body mass index (BMI [the weight in kilograms divided by height in meters squared - a screening tool to identify possible weight problems for adults that can be considered an alternative for direct measures of body fat]) surpasses $30 \mathrm{~kg} / \mathrm{m}^{2}$. As to the severity, the World Health Organization proposes the following classifications: obese class I, when BMI is between 30 and $34.9 \mathrm{~kg} / \mathrm{m}^{2}$; obese class II, when BMI is between 35 and $39.9 \mathrm{~kg} / \mathrm{m}^{2}$; and obese class III, when BMI surpasses $40 \mathrm{~kg} / \mathrm{m}^{2}{ }^{1}$

Besides the BMI range, it is important to consider the body-fat distribution. Excessive body-fat accumulation in the abdominal region is related to visceral fat deposition, which is strongly connected to cardiovascular risk factors: hyperglycemia; decrease in high density lipoprotein levels; and increase in triglyceride, apolipoprotein B, and low density lipoprotein serum levels. The waist circumference measurement is a method that has been widely used to evaluate abdominal adipose tissue accumulation, and an increase in this measurement represents a risk factor regardless of the morbidity and mortality of nontransmissible chronic diseases. ${ }^{1}$ 
The increase of adipose tissue alters the distribution of lipophilic drugs, whose accumulation in that tissue results in an increase in the necessary dosage to reach the same effectiveness and to extend the half-life, which, in turn, slows or diminishes their elimination. On the other hand, hydrophilic drugs weakly penetrate in the adipose tissue and, thus, they are more effective in individuals who are the ideal weight. ${ }^{3}$

Besides the psychosocial disorders, these patients are likely to have comorbidities associated with hypertension, hypertrophic cardiomyopathy, hyperlipidemia, diabetes, cholelithiasis, sleep apnea, hypoventilation, and degenerative arthritis. ${ }^{4}$ Obesity, therefore, is a disease that demands proper treatment and attention from health professionals. As a result of the great number of associated comorbidities, obese individuals normally make use of several medications with different pharmacokinetic and pharmacodynamic characteristics, such as different classes of antihypertensives (beta-blockers, angiotensinogen converting enzyme inhibitors), diuretics, antilipemics, and hypoglycemics.

Regarding the fact that all clinical treatment modalities for obesity are, in general, inefficient for the subgroup of patients with morbid obesity (BMI greater than $40 \mathrm{~kg} / \mathrm{m}^{2}$ ), the surgical approach must be taken into consideration. Despite its invasive nature, bariatric surgery has shown a consistent success rate (up to $50 \%$ reduction in weight of overweight patients) in maintaining weight reduction in the long term, ${ }^{4}$ and weight reduction is associated with the reduction of comorbidities like diabetes and hypertension. ${ }^{5}$ Far from being a cosmetic procedure, bariatric surgery does not involve surgical adipose tissue removal. Antiobesity surgeries may be didactically divided in procedures that: 1) limit the gastric capacity (the so-called restrictive surgeries); 2) interfere in the digestion (the malabsorptive procedures); or 3) are a combination of both techniques.

The following are basic strategies for surgical approach for obese patients that lead to confirmed long-lasting weight loss results: ${ }^{4}$

- Surgeries that promote a general intestinal malabsorption

o Jejunoileal bypass

o Jejunocolic bypass

- Gastric restrictive surgeries

o Vertical banded gastroplasty

o Gastric band

o Gastric stapling

o Sleeve gastrectomy

- Surgeries that combine gastric restriction with malabsorption

o Roux-en-Y gastric bypass (RYGB)
- Surgeries that combine maldigestion with malabsorption and gastric restriction

o Biliopancreatic diversion with partial gastrectomy

o Distal gastric bypass

o Duodenal switch.

Literature reviews have been made focusing on the medication distribution in obesity cases and the administration of nutrients and medications to bariatric surgery patients. However, the behavior of drugs used in severe comorbidities associated with obesity, such as major depression, cardiovascular problems, and endocrine disorders, has not been deeply studied in the postoperative period. ${ }^{6}$

As surgical procedures become an important approach for the treatment of obesity, it is important to report the observed alterations in pharmacokinetics of administered drugs in these patients.

\section{Restrictive procedures}

In restrictive procedures, by stapling the mucosa, a pouch (with a capacity of up to $30 \mathrm{~mL}$ ) is created in the gastric fundus with an opening in the base of approximately $1 \mathrm{~cm}$ in diameter through which food will pass and travel through the remaining gastrointestinal tract. This technique limits the amount of food intake and increases the gastric emptying time, keeping the sensation of satiety for longer.

The gastric band is also an option, and the method consists of placing a silicon ring around the upper part of the stomach, decreasing its diameter, thus reducing its storage capacity.

\section{Procedures that combine gastric restriction and malabsorption}

Malabsorptive procedures cause a greater and faster weight loss when compared with strictly restrictive methods. However, they are also associated with major complications, such as deficiency of nutrients and vitamins caused by the decrease in the absorptive area of the small intestine. ${ }^{?}$

Commonly known as biliopancreatic diversion, combined procedures were developed by Scopinaro of the University of Genoa in Italy. They consist of a partial gastrectomy of $80 \%$ (restrictive element) associated with the Roux-en-Y anastomosis with a long jejunal loop, and the entero-entero anastomosis located $50 \mathrm{~cm}$ from the ileocecal valve (disabsorptive element). ${ }^{4}$

The RYGB procedure is the most frequently used technique in the United States and is considered the gold standard of weight loss surgery. It consists of the creation of a proximal gastric pouch, which holds approximately $20 \mathrm{~mL}$, by stapling 
or transecting the stomach, and a gastro-jejunal anastomosis of this pouch in the Roux-en-Y., ${ }^{4,7}$

\section{Results of bariatric surgery in relation to the absorption and distribution of medications}

Some very punctual issues relating the absorption of drugs to bariatric surgery can be found in the literature, but little is known concerning the therapeutic conduct that health professionals must adopt when they face this situation. ${ }^{7}$

Drug solubility, the available surface area for absorption, and the blood flow in the gastrointestinal tract influence the absorption and the bioavailability of orally administered drugs. Procedures like the gastric bypass affect drug solubility and the absorptive area. ${ }^{8}$

The most common type of bariatric surgery is that which combines restrictive procedures, such as the reduction in the functional volume of the stomach, with malabsorptive procedures (eg, the connection of part of the duodenum and jejunum to the stomach). This technique culminates in the reduction of microvilosities surface area responsible for the great absorptive potential of the intestine. ${ }^{8}$

Medications that are soluble in an acid environment are more likely to be absorbed in the stomach, whereas those that are soluble in an alkaline environment are better absorbed in the small intestine. ${ }^{3}$ In patients who undergo gastric volume reduction surgeries, the hydrochloric acid production is decreased as well as the solubility and absorption of acid-soluble drugs. Likewise, the bariatric procedure that reduces the absorptive intestinal area leads to an alteration in absorption and distribution of the great majority of alkaline-soluble drugs. Moreover, the production of intestinal enzymes, in many cases important for the process of absorption of medications, is decreased. ${ }^{8}$

Therefore, drugs with long absorptive phases that remain in the intestine for extended periods are likely to present decreased bioavailability in patients who undergo malabsorptive procedures. ${ }^{8}$ Furthermore, the distribution of lipophilic drugs, like propofol, is altered due to the decrease in the fat mass in such patients. Drugs that would previously remain in the body for longer periods, deposited in the individual's large amount of adipose tissue, will have their half-life shortened, and if doctors do not pay attention to this particularity, cases of drug poisoning are likely to happen due to the administration of doses that would be suitable for obese patients.

Drugs that are orally administered must travel through the gastrointestinal tract, cross the cellular membranes until they reach the liver through portal circulation, and then be delivered into the systemic circulation. However, owing to the fact that only nonionized drugs cross the cellular membranes by passive diffusion, those that are ionized depend on active carriers to do so. Carrier proteins are located on the apical or basolateral surfaces of enterocytes, and they play an important role in the influx and efflux of substances. Groups of drugs have affinity for certain groups of carriers, and the frequently irregular distribution in the intestinal mucosa defines its absorption surface area. Surgical procedures, such as RYGB, shorten the small intestine and lead to the loss of a great part of active drug carriers located in that area, thus affecting its absorption. ${ }^{7}$

As a result, it is a medical duty to adjust the dose of each drug previously taken by an obese patient who has undergone a bariatric procedure so that the active principle in the proper intensity of each drug is kept, favoring efficient therapeutic maintenance and, thus, avoiding drug poisoning.

As this is an under-researched area in the medical literature, this article aims to summarize the study results that focus on the anatomo-physiological alterations that patients submitted to the surgical procedure undergo, and the consequences of these alterations for the use of drugs after surgery.

\section{Main drugs studied in the postoperative period in patients who have undergone bariatric surgery Antidepressants}

In a retrospective study involving 439 patients who underwent the RYGB procedure, Cunninghan et al reported that $23 \%$ of the patients showed an increase in use of antidepressant drugs after the surgery, $40 \%$ kept the use of the same class of antidepressants, $18 \%$ started on a new class of antidepressants, and only $16 \%$ showed a decrease in need of antidepressant treatment. ${ }^{9}$ Approximately $20 \%$ to $50 \%$ of bariatric surgery patients in the United States have a history of mood disorder. $^{10}$

Tricyclic antidepressants and selective serotonin receptor inhibitors are highly lipophilic drugs that are well-absorbed in the normal gastrointestinal tract. After gastric bypass surgery, the distribution volume of such drugs is smaller given the loss of adipose tissue.

In these patients, there is not only a reduction in the amount of absorbed drugs, but also a reduction in the time during which this absorption occurs. ${ }^{11}$

According to Hamad et al, patients using selective serotonin-receptor inhibitors are subject to the risk of a decrease in bioavailability of the drugs after the RYGB procedure, 
and, thus, they recommend a psychiatric follow-up after the surgery. ${ }^{11}$

Therefore, these patients constitute an important risk group owing to the decrease in the bioavailability of the drug they are using and the consequent development of depressive symptoms and suicidal tendencies. ${ }^{12}$

Once there is a shortage of research sources on the subject, health care professionals should always monitor their patients who undergo an antidepressant treatment, especially during the first month after the surgery, and be mindful of refractory symptoms, side effects, and accurate dosing and formulation, ${ }^{6,12}$ as most of these patients will keep the treatment after the bariatric procedure. ${ }^{10}$

\section{Antidiabetics}

Bariatric surgery results in significant weight loss and in the resolution or aggravation of type 2 diabetes mellitus cases. So far, only three randomized control trials showed the superiority of the surgery over the clinical treatment for type 2 diabetes. ${ }^{2,6}$

All current studies show a reduction in use of oral antidiabetics and insulin after the surgery in obese patients. ${ }^{2,6}$

Only metformin, the main oral hypoglycemic agent used in the treatment of type 2 diabetes mellitus, was deeply studied, and an increase in its absorption and in bioavailability was found in patients who underwent a RYGB procedure. ${ }^{2}$

The published studies thus far present methodological problems or lack details concerning the dosage of the studied drugs. $^{6}$

\section{Antilipemic drugs}

Segal et al investigated the use of antilipemic drugs in 6,235 bariatric surgery patients in a cohort study. The study reported that $59 \%$ of the nondiabetic patients and $54 \%$ of the diabetic patients showed a decrease in the need for the drug intake in the treatment of dyslipidemia 12 months after the surgery, thus indicating a substantial resolution of dyslipidemia after the surgical intervention. ${ }^{12}$

In a different study that involved ten patients who underwent the biliopancreatic diversion procedure, Skottheim et al reported an increase in the bioavailability of atorvastatin, indicating a great influence of the diverted small intestine. $^{13}$

Just like atorvastatin, simvastatin is a lipophilic drug; however, it is a prodrug that needs hydrolysis to form its active metabolite. The influence of the bariatric surgery on the first-passage metabolism of simvastatin is still unknown. ${ }^{6}$

\section{Antihypertensive agents}

In a study with 42 obese patients who were submitted to the RYGB procedure, Donadelli et al reported the decrease in all cardiovascular risk factors after the surgery. Besides, it was observed that $43 \%$ of the studied patients made use of antihypertensive drugs before the surgery and, 2 years after the procedure, only $17 \%$ of those patients continued to use them. ${ }^{14}$ The use of cardiotonic medications decreased in 54\% of patients, and of diuretics in $51 \%$ of patients, during this period. This decrease in use of antihypertensive drugs (diuretics, angiotensin-converting enzyme inhibitors, angiotensin receptor blockers, and beta-blockers) after bariatric surgery is also significant when compared with the conventional clinical treatment for obese patients. ${ }^{6}$

In the literature, only studies on the pharmacokinetics of beta-blockers after bariatric surgeries, such as atenolol and propranolol, could be found. ${ }^{6}$ Although the mentioned drugs are both alkaline agents, propranolol is lipophilic and atenolol is hydrophilic. It is known that the main altered parameters in a restrictive bariatric surgery are a decrease in gastric secretion of hydrochloric acid and an increase in the organ motility; hence, the gastric mucosa is covered by a less acidic solution - a fact that favors the absorption of such beta-blockers and other basic drugs in the region. However, the increase in stomach $\mathrm{pH}$ reduces the solubilization of alkaline drugs in the stomach, thus contributing to a better intestinal absorption of the drug. ${ }^{6}$

Therefore, if the use of beta-blockers after a bariatric procedure is necessary, a hydrophilic component such as atenolol should be prescribed. ${ }^{6}$

\section{Antibiotics}

Only two studies involving postoperative bariatric surgery and antibiotics can be found in the literature. ${ }^{15,16}$ One of these studies describes azithromycin, a macrolide antibiotic commonly prescribed in community-acquired pneumonia cases with the main absorption site in the duodenum. ${ }^{15}$ It is known that the gastric bypass procedure results in the reduction of the duodenal area; thus, a lower absorption of this drug in bariatric surgery patients is expected. Laboratory exams showed low plasma levels of azithromycin in gastric bypass patients when compared with a control group. Low plasma concentrations of the antibiotic in these individuals correspond to the consequent decrease of its concentration in tissues. ${ }^{16}$

Likewise, a study conducted by De Smet et al compared the plasma levels of moxifloxacin, a fluoroquinolone antibiotic used in acute respiratory infection cases, in patients 
who underwent the RYGB procedure and control groups. ${ }^{16}$ The researchers acknowledged the lack of references on the subject in the literature, and stated that, in most cases, the plasma concentration measurement of a drug to establish its therapeutic range is considered unnecessary once clinical parameters like blood pressure and glycemia can be more easily measured. However, the study concluded that there were no significant differences in the moxifloxacin plasma concentrations between bariatric surgery patients and the control group. ${ }^{17}$

\section{Analgesics}

In a normal population, the use of nonsteroidal antiinflammatory drugs (NSAIDs) is related to the onset of gastric ulcers in $15 \%$ to $30 \%$ of cases. Therefore, this class of medications is not recommended for bariatric surgery patients. ${ }^{7}$

Nonsteroidal anti-inflammatory drugs are weak acids that remain in molecular form in the gastric mucosa and ionize when in contact with the small intestine. In RYGB patients, the gastric $\mathrm{pH}$ is increased, making the medium more alkaline and allowing the ionization of the drug. As a consequence, there is a higher risk of the development of ulcers and mucosal erosions. ${ }^{7}$

\section{Oral contraceptives}

Mehri et al showed an increase in sexuality, fertility, and pregnancy rates in bariatric surgery patients. ${ }^{17}$ The use of oral contraceptives in these patients is not reliable owing to the lower absorption and bioavailability of these drugs after the surgical procedure. Alternative contraceptive methods should be considered. ${ }^{6}$

\section{Bariatric surgery and alcohol}

Candidates for bariatric surgery are part of an important risk group for alcohol and substance abuse. The psychological changes patients undergo after the surgery result in an increase in their sensitivity to alcohol. After each dose intake, these patients, due to their smaller stomach size, experience higher concentrations of ethanol than a normal individual. Furthermore, the faster gastric emptying, as a result of the surgery, allows for rapid alcohol absorption in the intestine. ${ }^{18}$

\section{Conclusion}

In most cases, after the performance of the surgical procedure and the resultant weight loss, the patient may develop some kind of nutritional deficiency, especially of vitamins and minerals. Furthermore, they may undergo alterations in the absorption and distribution of drugs once the body composition (in terms of water and fat) is altered, and may experience alterations in the production of gastric and intestinal enzymes due to the affected gastrointestinal area.

As a result of a restrictive procedure, the patient may undergo alterations in the gastric emptying time, $\mathrm{pH}$, and mucosal exposition, which will bring direct consequences concerning the absorption of orally administered drugs.

A decrease in drug absorption can be more frequently found in patients who are submitted to procedures that combine restrictive and malabsorptive techniques. The decreased functional length of the intestine and its consequent decreased absorptive surface lead to a lower absorption of extended release drugs as the drugs cross the entire gastrointestinal tract before their absorption is complete. In order to solve this problem, immediate release formulas should replace the conventional treatment with a resultant more frequent administration of such drugs. ${ }^{8}$

Drugs that are primarily absorbed in the stomach and duodenum will have their absorption decreased in patients who are submitted to combined procedures. Recent studies have investigated the possibility of compensatory absorption in other areas of the body, ${ }^{8}$ as well as hypertrophy of the intestinal mucosal, in an attempt to increase the intestinal absorptive area. ${ }^{6,12}$

Familiarity with the removed intestinal section during the surgery, as well as the pharmacokinetic properties of each prescribed drug, may help doctors understand the behavior of certain drugs postoperatively and prevent possible harm in their absorption. ${ }^{7}$

\section{Disclosure}

The authors report no conflicts of interest in this work.

\section{References}

1. Marcelino LF, Patrício ZM. [The complexity of obesity and life after bariatric surgery: a public health issue]. Cien Saude Colet. 2011;16(12):4767-4776. Portuguese.

2. Padwal RS, Gabr RQ, Sharma AM, et al. Effect of gastric bypass surgery on the absorption and bioavailability of metfotmin. Diabetes Care. 2011;34:1295-1300.

3. Sanches GD, Gazoni FM, Konishi RK, et al. [Intensive care of postoperative patients in bariatric surgery]. Rev Bras Ter Intensiva. 2007; 19(2):205-209. Portuguese.

4. Zilberstein B, Neto MG, Ramos AC. [The role of surgery in the treatment of obesity]. Rev Bras Med. 2002;59(4):258-264. Portuguese.

5. Csendes A1, Burdiles P, Papapietro K, Burgos AM. [Review of the results of medical and surgical treatment of morbid obesity]. Rev Med Chil. 2009;137(4):559-566. Spanish

6. Yska JP, van der Linde S, Tapper VV, et al. Influence of bariatric surgery on the use and pharmacokinetics of some major drug classes. Obes Surg. 2013;23(6):819-825. 
7. Smith A, Henriksen B, Cohen A. Pharmacokinetic considerations in Roux-en-Y gastric bypass patients. Am J Health Syst Pharm. 2011;68(23):2241-2247.

8. Miller AD, Smith KM. Medication and nutrient administration considerations after bariatric surgery. Am J Health Syst Pharm. 2006;63(19):1852-1857.

9. Cunninghan JL, Merrel CC, Sarr M, et al. Investigation of antidepressant medication usage after bariatric surgery. Obes Surg. 2012;22: 530-535.

10. Roerig JL, Steffen K, Zimmerman C, Mitchell JE, Crosby RD, Cao L. Preliminary comparison of sertraline levels in postbariatric surgery patients versus matched nonsurgical cohort. Surg Obes Relat Dis. 2012;8(1):62-66.

11. Hamad GG, Helsel JC, Perel JM, et al. The effect of gastric bypass on the pharmacokinetics of serotonin reuptake inhibitors. Am J Psychiatry. 2012;169(3):256-263.

12. Segal JB, Clark JM, Shore AD, et al. Prompt reduction in use of medications for comorbid conditions after bariatric surgery. Obes Surg. 2009;19(12):1646-1656.
13. Skottheim IB, Jakobsen GS, Stormark K, et al. Significant increase in systemic exposure of atorvastatin after biliopancreatic diversion with duodenal switch. Clin Pharmacol Ther. 2010;87(6):699-705.

14. Donadelli SP, Salgado W Jr, Marchini JS, et al. Change in predicted 10-year cardiovascular risk following Roux-en-Y gastric bypass surgery: Who benefits? Obes Surg. 2011;21(5):569-573.

15. Padwal RS, Ben-Eltriki M, Wang X, et al. Effect of gastric bypass surgery on azithromycin oral bioavailability. J Antimicrob Chemother. 2012;67(9):2203-2206.

16. De Smet J, Colin P, De Paepe P, et al. Oral bioavailability of moxifloxacin after Roux-en-Y gastric bypass surgery. J Anrimicrob Chemother. 2012;67(1):226-229.

17. Merhi ZO. Impact of bariatric surgery on female reproduction. Fertil Steril. 2009;92(5):1501-1508.

18. Heinberg LJ, Ashton K, Coughlin J. Alcohol and bariatric surgery: review and suggested recommendations for assessment and management. Surg Obes Relat Dis. 2012;8(3):357-363.

\section{Publish your work in this journal}

The International Journal of General Medicine is an international, peer-reviewed open-access journal that focuses on general and internal medicine, pathogenesis, epidemiology, diagnosis, monitoring and treatment protocols. The journal is characterized by the rapid reporting of reviews, original research and clinical studies across all disease areas.
A key focus is the elucidation of disease processes and management protocols resulting in improved outcomes for the patient. The manuscript management system is completely online and includes a very quick and fair peer-review system. Visit http://www.dovepress.com/ testimonials.php to read real quotes from published authors. 